\title{
Diseño de un amplificador lineal de microondas para portadoras de Banda-X
}

\section{(Design of a linear microwave amplifier for X-Band carriers)}

\author{
Cristhian Castro-Peñaherrera ${ }^{1}$, Carlos Alberto Serra Jiménez ${ }^{2}$
}

\begin{abstract}
Resumen:
En el presente artículo se exponen los resultados obtenidos en el diseño de un amplificador lineal de microondas para portadoras de banda X. Fundamentalmente se destacan los aportes realizados en las técnicas de implementación de circuitos amplificadores de alta frecuencia basados en transistores de tecnología HEMT de GaN; así como la elaboración de las líneas de polarización y puertos de adaptación, control de estabilidad y ganancias en el rango de frecuencias deseado. Se debe destacar la utilización de herramientas de software libre para la caracterización del transistor mediante sus parámetros S y la geometría de las líneas de transmisión del circuito.
\end{abstract}

Palabras clave: líneas de transmisión; ROE; coeficiente de reflexión; parámetros S.

\begin{abstract}
:
In the present article are exposed the results obtained in the design of a linear microwave amplifier for X-band carriers. It fundamentally shows the contributions made in the techniques of implementation of high frequency amplifier circuits based on transistors of GaN HEMT technology, as well as the elaboration of polarization lines and adaptation ports, stability control and gains in the desired frequency range. It should be noted the use of free software tools for the characterization of the transistor through its $\mathrm{S}$ parameters and the geometry of the transmission lines of the circuit.
\end{abstract}

Keywords: transmission lines; SWR; reflection coefficient; S-parameters.

\section{Introducción}

Los amplificadores de potencia de RF son usualmente la última etapa de un sistema de comunicación inalámbrico justo antes de las antenas, al ser este un dispositivo electrónico es mayoritariamente el que condiciona la potencia de transmisión, consumo de energía y tamaño físico del equipo, de allí la constante importancia en el desarrollo de técnicas para mejorar el rendimiento de este tipo de dispositivos (Hu, Qian, \& Wang, 2015).

Uno de los sectores en los cuales mayoritariamente se han desarrollado aplicaciones en banda-X, es el aeroespacial; en la transmisión de datos de satélites de observación terrestre, o en sensores que utilizan radares de apertura sintética, estos instrumentos a bordo de aeronaves espaciales requieren ser más eficientes en cuanto a consumo de potencia por ancho de banda y tamaño (Resca, y otros, 2014). La tecnología de fabricación de transistores de potencia para microondas ha conseguido la construcción de amplificadores pequeños y con grandes niveles de ganancia. Una de las principales tecnologías de fabricación de transistores para microondas con estas características son los HEMT de GaN. Su eficiencia radica en su alta impedancia y baja capacitancia de los puertos de entrada y salida lo cual permite que los dispositivos

\footnotetext{
1 Universidad Politécnica de Valencia, Valencia - España (cricaspe@upv.edu.es).

2 Universidad Técnica de Ambato, Ambato - Ecuador (carlos.serra@uta.edu.ec).
} 
trabajen en un amplio rango de frecuencias con altos niveles de potencia de salida y con un consumo eléctrico relativamente bajo (Yarman \& Ejaz, 2015).

Las técnicas de diseño y topologías de amplificadores de radiofrecuencia presentan optimización en sus parámetros según: la aplicación, la tecnología de fabricación del elemento activo, el ancho de banda y la frecuencia de operación. Para aplicaciones con tecnología wi-fi o bluetooth el diseño se optimiza para obtener la máxima ganancia con muy poco ruido (Köprü, Kuntman , \& Yarman, 2013). Cuando se trata de diseñar amplificadores de alta potencia es común diseñarlos con la técnica de Push-Pull para adaptar adecuadamente los puertos de entrada y salida (Smith \& Cripps, 2016). Otro de los escenarios de diseño se plantea en función del ancho de banda y la máxima transferencia de potencia (Setiawan, Latin, \& Alam, 2017), en este escenario se pide elaborar un diseño simplificado, estable y adaptado a la transmisión de tonos puros en banda $X$, con la consecuente disminución en costos y en lo posible con el uso de elementos locales.

El objetivo de este trabajo es diseñar y construir un amplificador de potencia para un transmisor que trabaja como una fuente de referencia, el cual es utilizado para calibrar los sistemas de recepción satelital del Instituto Espacial Ecuatoriano (IEE), para lo cual únicamente se requiere enviar señales portadoras en banda $X$. Si se tiene en cuenta esta consideración, la topología más eficiente es un amplificador lineal clase A con excursión completa en todo el rango de la señal sinusoidal (Instituto Espacial Ecuatoriano, 2016).

Para la realización del diseño se comienza por la elección del transistor de acuerdo con las especificaciones del transmisor, al transistor se lo modela como una red de dos puertos caracterizada por su matriz de parámetros de dispersión, luego se calcula la estabilidad de la red y la máxima ganancia de potencia disponible en el rango de frecuencias de trabajo (Froehner, 1967).

Mediante la red de polarización se fija el punto de operación del transistor en el valor especificado por la matriz de parámetros-S, posteriormente se calculan los parámetros-S en la nueva red que contiene al transistor, las redes de polarización y el sustrato. Con los valores de esta nueva red se diseña las redes de adaptación de puertos de entrada y salida para conseguir que la relación de onda estacionaria sea cercana a 1:1. Para la construcción de la red se utilizan líneas de transmisión y elementos concentrados con empaquetado físico mucho menor a la frecuencia de operación (Virdee, Virdee, \& Banyamin, 204)

\section{Metodología}

Un amplificador de RF básicamente se representa caracterizando el elemento activo como una matriz de parámetros de dispersión en una red de dos puertos ubicado entre sus redes de adaptación de entrada y salida. En la Figura 1 se observa el esquema de análisis del amplificador de microondas.

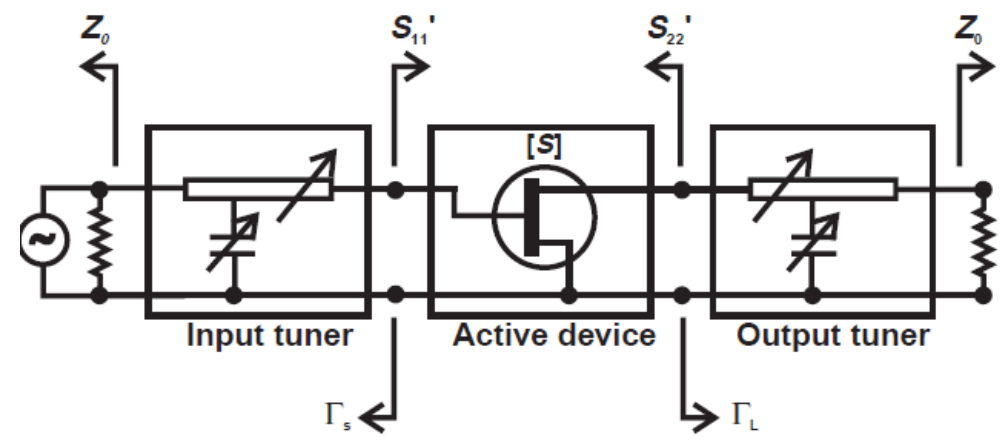

Figura 1. Esquema de análisis de un amplificador de microondas (Cripps, 2006) 
Del esquema de análisis de un amplificador de microondas se deduce que su diseño consiste en calcular los valores de las impedancias de entrada y salida, e implementar adecuadamente la red de polarización que mantenga al transistor en el punto de operación seleccionado.

El parámetro $S_{11}^{\prime}$ representa la potencia de la onda incidente en la entrada del puerto 1 y $S_{22}^{\prime}$ es la porción de onda reflejada por la carga hacia la entrada del puerto 2. Estas dos magnitudes están directamente relacionadas con los coeficientes de reflexión en la entrada $S_{11}$ y coeficiente de transmisión $S_{21}$, que a su vez están relacionados con los coeficientes de reflexión de entrada y salida (Soler, 2015). Por lo tanto, la relación entre estas magnitudes permite calcular los valores de impedancias de entrada y salida que posibiliten la máxima transferencia de potencia, ganancia y ancho de banda adecuados.

\subsection{Caracterización del elemento activo}

Para el diseño del amplificador se persigue que este trabaje con un ancho de banda de $400 \mathrm{MHz}$ a partir de los $8.0 \mathrm{GHz}$ hasta $8.4 \mathrm{GHz}$, con una ganancia de $15 \mathrm{~dB}$ y acoplamiento de puertos de entrada y salida $1: 1$ referida a $50 \Omega$.

Para alcanzar el rendimiento deseado la tecnología de dispositivos HEMT de GaN es la más adecuada por su gran ancho de banda de funcionamiento y potencia (Yarman \& Ejaz, 2015). De los dispositivos disponibles para la frecuencia de operación se analiza: el factor K de estabilidad, MAG (Máxima Ganancia Disponible, Maximum Gain Available) y PAE (Eficiencia de la Potencia Agregada, Power Added Efficiency). El transistor seleccionado es el TGF2023-2-01 fabricado por Qorvo en tecnología de 1.25mm GaN en SiC HEMT, cuyas características principales se observan en la Tabla 1.

Tabla 1. Características del TGF2023-2-01

\begin{tabular}{|c|c|}
\hline Rango de frecuencia & $\mathrm{DC}-18 \mathrm{GHz}$ \\
\hline Alimentación & $\mathrm{VD}=12-32 \mathrm{~V}, \mathrm{IDQ}=25-125 \mathrm{~mA}$ \\
\hline Rango de temperatura & $-65 \mathrm{a} 150^{\circ} \mathrm{C}$ \\
\hline Ganancia de potencia nominal & $18 \mathrm{~dB}$ a $3 \mathrm{GHz}$ \\
\hline Potencia de saturación (Psat) & $38 \mathrm{~dB} \mathrm{a} \mathrm{3} \mathrm{GHz}$ \\
\hline Máxima PAE & $71.6 \%$ \\
\hline
\end{tabular}

\subsection{Análisis de estabilidad}

Para realizar el análisis del elemento activo y en general para el diseño y simulación del amplificador se ha optado por utilizar herramientas de software libre, el programa seleccionado es Qucs (Quite universal circuit simulator), que posee herramientas de soporte para diseño de líneas de transmisión y en general circuitos de microondas (Qucs, 2017). En el espacio de trabajo de Qucs se inserta una red dos puertos caracterizada por el archivo " $s 2 p$ " que es la matriz de parámetros de dispersión del dispositivo proporcionado en la documentación del fabricante.

La estabilidad de un elemento se define como la oposición del mismo a oscilaciones, esta característica fundamental en un amplificador queda definida por la Ecuación 1, denominada factor de estabilidad de Rollet. El simultáneo cumplimiento de ambas condiciones indica que la red es estable, caso contrario la red sería potencialmente inestable para valores fuera de este rango.

$$
\begin{gathered}
k=\frac{1-\left|S_{11}\right|^{2}-\left|S_{22}\right|^{2}+|\Delta|^{2}}{2\left|S_{12} S_{21}\right|}>1 \\
\Delta=S_{11} S_{22}-S_{12} S_{21}<1
\end{gathered}
$$

Al realizar la simulación mediante un barrido en frecuencia desde $1 \mathrm{GHz}$ hasta $18 \mathrm{GHz}$ de los parámetros-S en el punto de operación $V_{G}=-3.6 \mathrm{Vdc}, V_{D}=28 \mathrm{Vdc} y \mathrm{I}_{D}=$ 
$125 \mathrm{~mA}$, se obtiene la curva mostrada en la Figura 2, de la cual se extrae que el transistor es incondicionalmente estable para el rango de frecuencias comprendido entre los $6.5 \mathrm{GHz}$ y $15.5 \mathrm{GHz}$. El hecho de tener estabilidad incondicional en el rango de diseño nos permite construir redes de acoplamiento que consigan la máxima transferencia de potencia y ganancia.

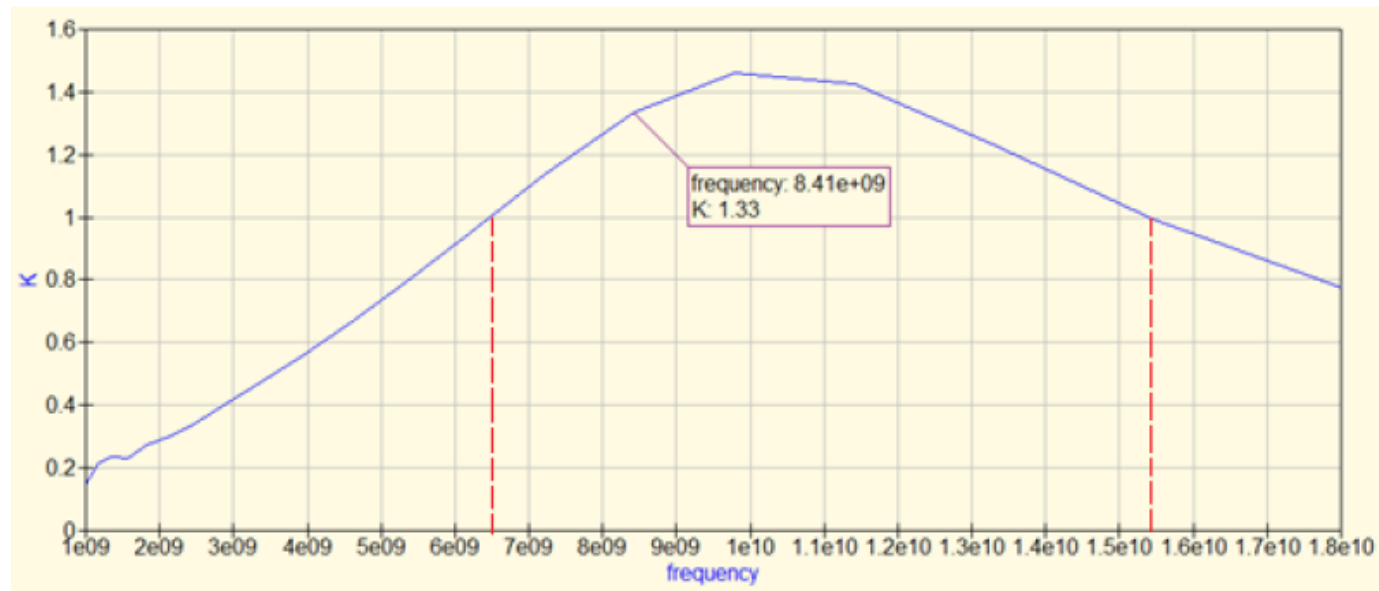

Figura 2. Análisis de estabilidad TGF2023-2-01

\subsection{Análisis de la máxima ganancia de potencia}

Ahora que conocemos que el transistor es incondicionalmente estable para la franja de frecuencias de diseño, es importante conocer la máxima ganancia disponible que el transistor puede lograr entregar hacia una carga completamente adaptada. Utilizando las ecuaciones 2 y 3 se calcula la máxima ganancia disponible.

$$
\begin{gathered}
M A G=10 \log \left(\frac{\left|S_{21}\right|}{\left|S_{12}\right|}\left|k \pm \sqrt{k^{2}-1}\right|\right) \\
B_{1}=10 \log \left(\frac{\left|S_{21}\right|}{\left|S_{12}\right|}\left|k \pm \sqrt{k^{2}-1}\right|\right)
\end{gathered}
$$

El término $B_{1}$ determina el signo en el radical de la ecuación de MAG, si su signo es positivo se utilizará (-) antes del radical y si $B_{1}$ es negativo entonces se utiliza $(+)$ en el radical. Esta vez, para realizar el cálculo de la ganancia, se parametriza el bloque de simulación para que realice un barrido desde los $6 \mathrm{GHz}$ a $12 \mathrm{GHz}$ con lo que se obtiene mejor resolución en el rango en el cual deseamos conocer el comportamiento de la ganancia. En la figura 3 se observa la gráfica del comportamiento de ganancia máxima en función de la frecuencia.

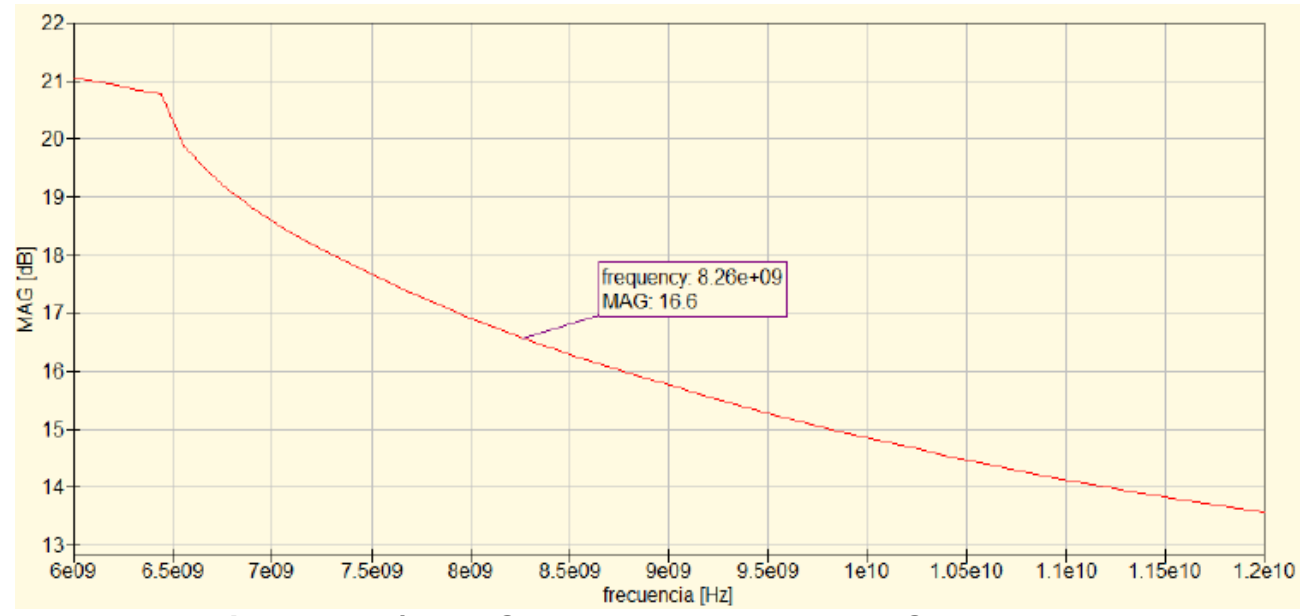

Figura 3. Máxima Ganancia Disponible del TGF2023-2-01 
La ganancia del amplificador en el rango de frecuencias requerido cumple con las especificaciones. Además, habrá que recordar que también existirán pérdidas en las redes de adaptación, por lo cual (Sayre, 2001) recomienda reducir en un 10\% la potencia calculada. Con esta consideración la ganancia del amplificador final sería de $14.94 \mathrm{~dB}$ en la frecuencia central de diseño, por lo cual el diseño de las redes de adaptación perseguirá la máxima ganancia disponible del elemento activo.

\subsection{Vías de polarización}

El diseño de esta etapa del circuito fija el punto de operación seleccionado, en este caso $V_{G}=-3.6 \mathrm{Vdc}, V_{D}=28 \mathrm{Vdc}$ e $I_{d}=125 \mathrm{~mA}$ que son los valores dados en los parámetros de medida de parámetros-S por el fabricante. El transistor seleccionado es un HEMT, su esquema de polarización se presenta en la figura 4. El objetivo de esta red es proporcionar al dispositivo los niveles de DC adecuados e impedir que las oscilaciones de RF afecten a la fuente de polarización (Glover, Penoock, \& Shepherd, 2005). La red se comporta como un corto circuito para las componentes de corriente continua y es un circuito abierto para la señal de alta frecuencia.

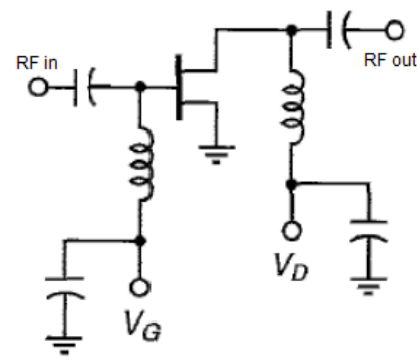

Figura 4. Esquema de polarización de un HEMT

En la Figura 5 se presenta la red de polarización. Para su diseño se tomó de referencia el propio esquema de polarización de HEMT, en el cual se observan los capacitores de bypass de entrada y salida de RF que evitan el paso de cualquier componente continua parásita que pudiera inducirse a la entrada o salida del transistor, el capacitor de carga de DC que soporta la demanda inmediata de corriente del transistor ocasionada por la propia conmutación y la bobina de choque para evitar posibles acoplamientos de la señal de alta frecuencia.

El capacitor de carga se diseñó mediante cuatro capacitores en paralelo de valor sucesivo para cubrir un gran ancho de banda y capacidad de respuesta a requerimientos transitorios de corriente, el valor de la bobina de choque se obtiene al calcular la reactancia inductiva $X_{L}=\omega L$. Es importante en este punto tener en cuenta los valores comerciales de bobinas disponibles en el mercado, en este caso se seleccionó una inductancia de $L=47 \mathrm{mH}$ que nos brinda una impedancia de $2.43 \mathrm{M} \Omega$ a la frecuencia de $8.260 \mathrm{GHz}$.

El capacitor de bypass se calcula utilizando la reactancia capacitiva $X_{C}=1 / \omega C$, con $C=1 p F$ se obtiene una impedancia de $19.268 \Omega$. En este caso el elemento va a trabajar en la zona de RF y a la frecuencia de operación las dimensiones de empaquetado del elemento y el sustrato sobre el cual se va a montar juegan un importante papel para su elección. En el trabajo de (Perez \& Jaramillo, 2012) se recomienda que para trabajar en FR4 con frecuencias superiores a $7.5 \mathrm{GHz}$ se debe utilizar empaquetado $0201 \mathrm{SMD}$.

En el diseño de la red se observan dos líneas de transmisión micro-tira MS3 y MS1; su función es adaptar las impedancias de los elementos discretos con la impedancia del transistor. El diseño de MS3 que trabaja en la zona de polarización debe verse como circuito abierto para altas frecuencias, es decir una impedancia muy superior a los $50 \Omega$ y fase $\lambda / 2$, mientras que para MS1 que trabaja en la zona de RF debe verse como un corto 
circuito a altas frecuencias, por lo tanto, la impedancia de este debe ser de $50 \Omega$ con fase $\lambda / 4$. Los valores de largo y ancho de pista dependen del sustrato sobre el cual se van a construir y se obtuvieron con la herramienta de síntesis de Líneas de Transmisión de Qucs.

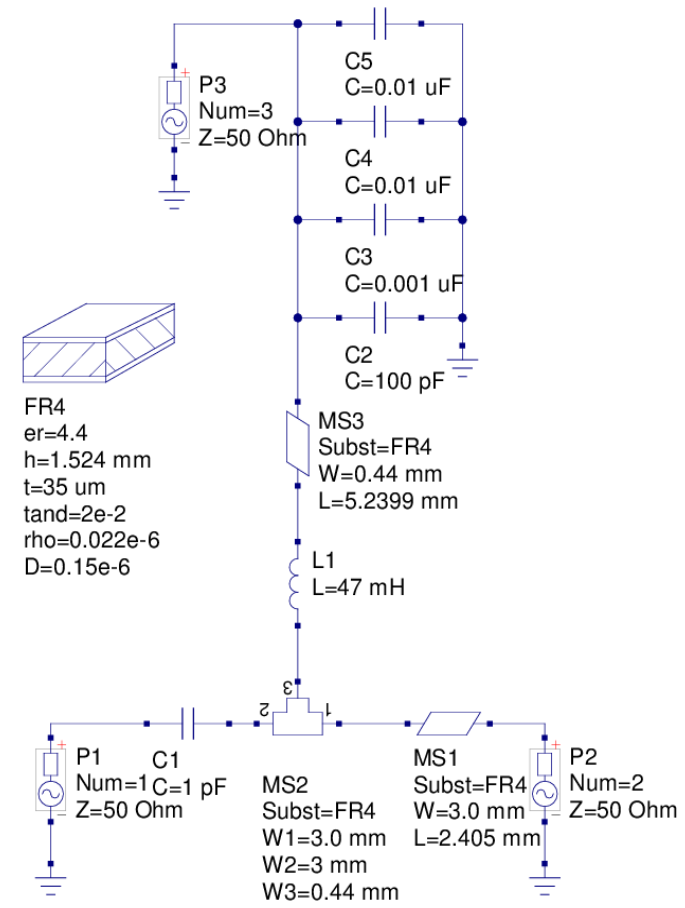

Figura 5. Red de polarización

La red de adaptación se encuentra caracterizada por los valores de elementos discretos y distribuidos por lo que se puede obtener la matriz de parámetros-S de esta red. En la Figura 6 se observa la simulación de la red de polarización con barrido de parámetros $S$ desde $1 \mathrm{GHz}$ hasta $12 \mathrm{GHz}$. En este rango la potencia de la señal que recibe el puerto P2 del puerto P1 tendrá una atenuación de $-0.179 \mathrm{~dB}$, además se observa que mientras la frecuencia de la señal disminuye o aumenta en el rango de diseño, esta sufre una atenuación considerable. La señal que viaja desde el puerto P3 hacia P1 y P2 recibe una atenuación de $-221 d B$ por todo el rango de altas frecuencias, lo cual deja pasar únicamente las componentes de DC.

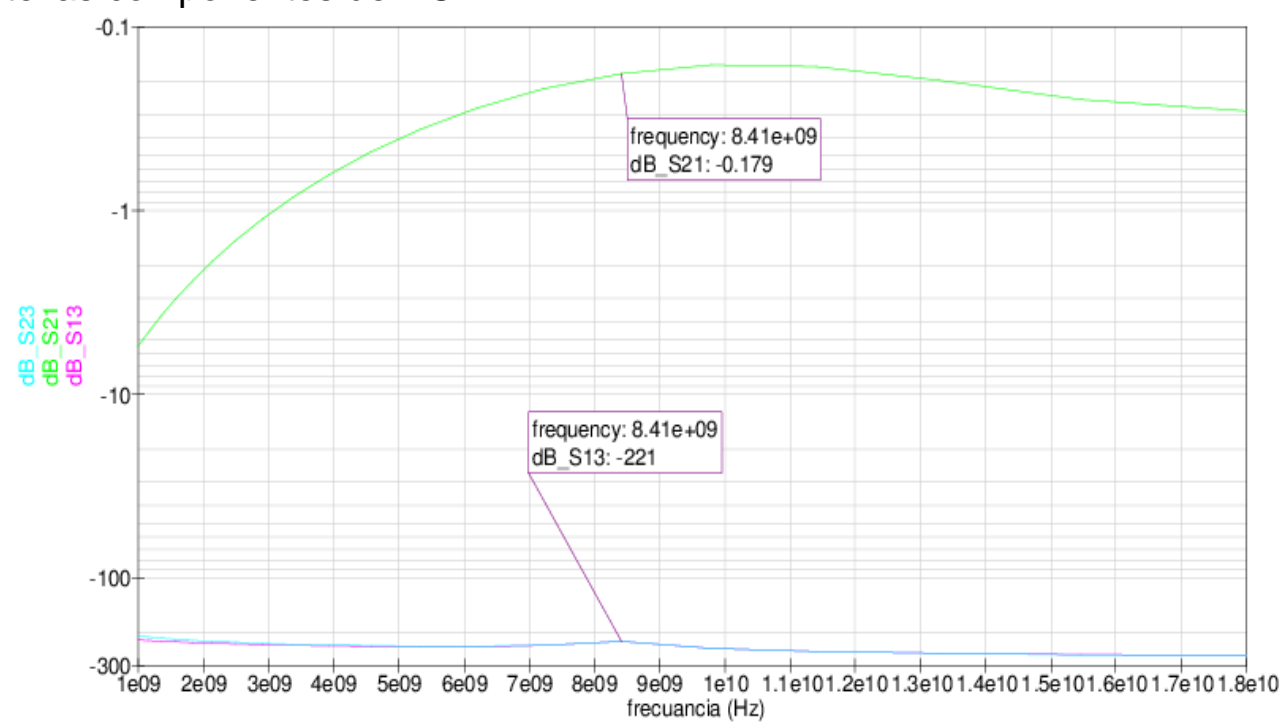

Figura 6. Parámetros-S de la red de polarización 


\subsection{Redes de adaptación}

Al momento de seleccionar la forma en la cual se va a diseñar las redes de adaptación se debe observar el valor del parámetro $S_{12}$, si este es igual a 0 entonces se puede realizar una adaptación unilateral o caso contrario se debe aplicar adaptación bilateral conjugada, realizando un barrido en frecuencia ajustando los parámetros para tener una resolución adecuada al rango de interés. Los resultados obtenidos se muestran en la Tabla 2, en la cual se observa que el parámetro $S_{12}$ en la frecuencia central de diseño $8.260 \mathrm{GHz}$ es $0.0242 \angle 13.3^{\circ}$.

Tabla 2. Parámetros $\mathrm{S}$ del amplificador, precálculo de la red de adaptación

\begin{tabular}{|c|c|c|c|c|}
\hline Frecuencia $[\mathrm{Hz}]$ & $\mathrm{S}[1,1]$ & $\mathrm{S}[1,2]$ & $\mathrm{S}[2,1]$ & $\mathrm{S}[2,2]$ \\
\hline $7.170 \mathrm{E}+09$ & $0.54 / 30.4^{\circ}$ & $0.172 /-71.4^{\circ}$ & $2.21 /-116^{\circ}$ & $0.426 / 94.8^{\circ}$ \\
\hline $7.300 \mathrm{E}+09$ & $0.536 / 27.1^{\circ}$ & $0177 /-74.6^{\circ}$ & $2.17 /-120^{\circ}$ & $0.431 / 91.5^{\circ}$ \\
\hline $7.430 \mathrm{E}+09$ & $0.533 / 23.7^{\circ}$ & $0.182 /-77.9^{\circ}$ & $2.13 /-124^{\circ}$ & $0.436 / 88.2^{\circ}$ \\
\hline $7.560 \mathrm{E}+09$ & $0.529 / 20.3^{\circ}$ & $0.187 /-81.3^{\circ}$ & $2.1 /-128^{\circ}$ & $0.441 / 84.9^{\circ}$ \\
\hline $7.700 \mathrm{E}+09$ & $0.524 / 16.8^{\circ}$ & $0.193 /-84.7^{\circ}$ & $2.07 /-132^{\circ}$ & $0.445 / 81.5^{\circ}$ \\
\hline $7.830 \mathrm{E}+09$ & $0.518 / 13.2^{\circ}$ & $0.198 /-88.3^{\circ}$ & $2.04 /-136^{\circ}$ & $0.449 / 78.2^{\circ}$ \\
\hline $7.970 \mathrm{E}+09$ & $0.513 / 9.51^{\circ}$ & $0.205 /-92^{\circ}$ & $2.01 /-140^{\circ}$ & $0.454 / 74.8^{\circ}$ \\
\hline $8.120 \mathrm{E}+09$ & $0.507 / 5.75^{\circ}$ & $0.211 /-95.7^{\circ}$ & $1.98 /-145^{\circ}$ & $0.458 / 71.4^{\circ}$ \\
\hline $8.260 \mathrm{E}+09$ & $0.5 / 1.88^{\circ}$ & $0.217 /-99.6^{\circ}$ & $1.95 /-149^{\circ}$ & $0.462 / 67.9^{\circ}$ \\
\hline $8.410 \mathrm{E}+09$ & $0.493 /-2.11^{\circ}$ & $0.224 /-104^{\circ}$ & $1.93 /-153^{\circ}$ & $0.466 / 64.4^{\circ}$ \\
\hline $8.560 \mathrm{E}+09$ & $0.486 /-6.23^{\circ}$ & $0.232 /-108^{\circ}$ & $1.9 /-158^{\circ}$ & $0.47 / 60.9^{\circ}$ \\
\hline $8.710 \mathrm{E}+09$ & $0.478 /-10.5^{\circ}$ & $0.24 /-112^{\circ}$ & $1.88 /-163^{\circ}$ & $0.473 / 57.3^{\circ}$ \\
\hline $8.870 \mathrm{E}+09$ & $0.469 /-14.9^{\circ}$ & $0.248 /-116^{\circ}$ & $1.86 /-167^{\circ}$ & $0.477 / 53.6^{\circ}$ \\
\hline $9.030 \mathrm{E}+09$ & $0.461 /-19.5^{\circ}$ & $0.256 /-121^{\circ}$ & $1.84 /-172^{\circ}$ & $0.48 / 49.7^{\circ}$ \\
\hline $9.190 \mathrm{E}+09$ & $0.452 /-24.2^{\circ}$ & $0.265 /-125^{\circ}$ & $1.82 /-177^{\circ}$ & $0.483 / 45.9^{\circ}$ \\
\hline $9.360 \mathrm{E}+09$ & $0.443 /-29.1^{\circ}$ & $0.275 /-130^{\circ}$ & $1.8 / 178^{\circ}$ & $0.487 / 41.8^{\circ}$ \\
\hline $9.520 \mathrm{E}+09$ & $0.435 /-34.2^{\circ}$ & $0.285 /-135^{\circ}$ & $1.78 / 172^{\circ}$ & $0.49 / 37.6^{\circ}$ \\
\hline $9.700 \mathrm{E}+09$ & $0.426 /-39.5^{\circ}$ & $0.296 /-140^{\circ}$ & $1.77 / 167^{\circ}$ & $0.492 / 33.2^{\circ}$ \\
\hline
\end{tabular}

La adaptación bilateral conjugada consiste en hacer que $\Gamma_{S}=\Gamma_{I N}^{*}$ y $\Gamma_{L}=\Gamma_{\text {OUT }}^{*}$, como se muestran en las ecuaciones 4 y 5 , luego se calculan los coeficientes de reflexión de fuente $\Gamma_{M S}$ y carga $\Gamma_{M L}$ mostrados en las ecuaciones 6 y 7 .

$$
\begin{gathered}
\Gamma_{I N}=S_{11}+\frac{S_{12} S_{21} \Gamma_{L}}{1+S_{22} \Gamma_{L}} \\
\Gamma_{\text {out }}=S_{22}+\frac{S_{12} S_{21} \Gamma_{S}}{1+S_{11} \Gamma_{S}} \\
\Gamma_{M S}=\frac{B_{1} \pm \sqrt{\left|B_{1}\right|^{2}-4\left|C_{1}\right|^{2}}}{2 C_{1}} \\
\Gamma_{M L}=\frac{-B_{2} \pm \sqrt{\left|B_{2}\right|^{2}-4\left|C_{2}\right|^{2}}}{2 C_{2}}
\end{gathered}
$$


Donde:

$B_{1}=1+\left|S_{11}\right|^{2}-\left|S_{22}\right|^{2}-|\Delta|^{2}$

$B_{2}=1+\left|S_{22}\right|^{2}-\left|S_{11}\right|^{2}-|\Delta|^{2}$

$C_{1}=S_{11}-\Delta S_{22}^{*}$

$C_{2}=S_{22}-\Delta S_{11}^{*}$

Ahora con los valores de parámetros-S mostrados en la Tabla 2 y las ecuaciones anteriores se calcula el valor de los coeficientes de reflexión de carga y fuente. Estos valores se convierten en impedancia y finalmente se aplican a la red de adaptación. En la Tabla 3, se observa el cálculo de coeficientes de reflexión, VSWR e Impedancias.

Tabla 3. Parámetros-S del amplificador con red de adaptación

\begin{tabular}{|c|c|c|c|c|c|c|}
\hline Frecuencia [Hz] & $z_{S}[\Omega]$ & $z_{L}[\Omega]$ & $S W R_{\text {in }}$ & $S W R_{\text {out }}$ & $\Gamma_{s}$ & $\Gamma_{L}$ \\
\hline $7.040 \mathrm{E}+09$ & $38.7 / 12^{\circ}$ & $49.9 / 1.33^{\circ}$ & 1.4 & 1.02 & $0.165 / 141^{\circ}$ & $0.0116 / 94.1^{\circ}$ \\
\hline $7.170 \mathrm{E}+09$ & $40.4 / 11.5^{\circ}$ & $50 / 1.42^{\circ}$ & 1.34 & 1.03 & $0.146 / 137^{\circ}$ & $0.0124 / 90.2^{\circ}$ \\
\hline $7.300 \mathrm{E}+09$ & $42.1 / 10.8^{\circ}$ & $50.1 / 1.53^{\circ}$ & 1.29 & 1.03 & $0.128 / 133^{\circ}$ & $0.0134 / 85.8^{\circ}$ \\
\hline $7.434 \mathrm{E}+09$ & $43.8 / 9.93^{\circ}$ & $50.2 / 1.67^{\circ}$ & 1.24 & 1.03 & $0.109 / 127^{\circ}$ & $0.0147 / 80.8^{\circ}$ \\
\hline $7.560 \mathrm{E}+09$ & $45.4 / 9.04^{\circ}$ & $50.5 / 1.94^{\circ}$ & 1.2 & 1.04 & $0.0925 / 122^{\circ}$ & $0.0175 / 75^{\circ}$ \\
\hline 7.709E+09 & $47.7 / 7.95^{\circ}$ & $51.1 / 1.93^{\circ}$ & 1.16 & 1.04 & $0.0734 / 109^{\circ}$ & $0.02 / 57.7^{\circ}$ \\
\hline 7.834E+09 & $49.8 / 5.99^{\circ}$ & $517 / 1.69^{\circ}$ & 1.11 & 1.05 & $0.0524 / 92.3^{\circ}$ & 0.0222 / 41.7' \\
\hline $7.970 \mathrm{E}+09$ & $51.5 / 3.3^{\circ}$ & $52.5 / 1.11^{\circ}$ & 1.07 & 1.05 & $0.0332 / 63^{\circ}$ & $0.0262 / 21.7^{\circ}$ \\
\hline $8.120 \mathrm{E}+09$ & $52.21 /-0.00349^{\circ}$ & $53.5 /-0.279^{\circ}$ & 1.04 & 1.07 & $0.0213 /-0.0819^{\circ}$ & $0.0343 /-4.06^{\circ}$ \\
\hline $8.260 E+09$ & $49.4 / 4.14^{\circ}$ & $54.5 /-3.89^{\circ}$ & 1.08 & 1.12 & $0.0366 /-99.2^{\circ}$ & $0.0552 /-37.9^{\circ}$ \\
\hline $8.410 \mathrm{E}+09$ & $37.2 /-6.58^{\circ}$ & $52.5 /-13.7^{\circ}$ & 1.37 & 1.28 & $0.157 /-159^{\circ}$ & $0.1221 /-78.5^{\circ}$ \\
\hline $8.564 \mathrm{E}+09$ & $15.3 / 9.64^{\circ}$ & $374 / 42.4^{\circ}$ & 3.33 & 1.96 & $0.538 / 174^{\circ}$ & $0.324 /-119^{\circ}$ \\
\hline $8.711 \mathrm{E}+09$ & $16.8 / 31.7^{\circ}$ & $24.1 / 25.2^{\circ}$ & 3.61 & 2.41 & $0.566 / 158^{\circ}$ & $0.414 /-152^{\circ}$ \\
\hline $8.870 \mathrm{E}+09$ & $39.4 / 8.68^{\circ}$ & $36.5 /-0.979^{\circ}$ & 1.33 & 1.37 & $0.141 / 148^{\circ}$ & $0.157 /-177^{\circ}$ \\
\hline $9.030 \mathrm{E}+09$ & $46.8 / 3.19^{\circ}$ & $46.2 / 1.27^{\circ}$ & 1.09 & 1.09 & $0.0436 / 140^{\circ}$ & $0.0412 / 164^{\circ}$ \\
\hline $9.194 \mathrm{E}+09$ & $47.6 / 2.94^{\circ}$ & $49 / 0.643^{\circ}$ & 1.07 & 1.02 & $0.0358 / 134^{\circ}$ & $0.0114 / 151^{\circ}$ \\
\hline $9.360 \mathrm{E}+09$ & $47.9 / 3.11^{\circ}$ & $49.7 / 0.252^{\circ}$ & 1.07 & 1.01 & $0.0348 / 129^{\circ}$ & $0.00339 / 140^{\circ}$ \\
\hline $9.520 \mathrm{E}+09$ & $48.2 / 3.08^{\circ}$ & $49.9 / 0.0977^{\circ}$ & 1.07 & 1 & $0.0324 / 124^{\circ}$ & $0.00112 / 131^{\circ}$ \\
\hline $9.791 \mathrm{E}+09$ & $48.6 / 2.9^{\circ}$ & $50 / 0.0517^{\circ}$ & 1.06 & 1 & $0.0292 / 120^{\circ}$ & $0.000539 / 123^{\circ}$ \\
\hline $9.870 \mathrm{E}+09$ & $48.9 / 2.68^{\circ}$ & $50 / 0.049^{\circ}$ & 1.05 & 1 & $0.0258 / 116^{\circ}$ & $0.000479 / 117^{\circ}$ \\
\hline $1.000 \mathrm{E}+10$ & $49.2 / 2.39^{\circ}$ & $50 / 0.0616^{\circ}$ & 1.05 & 1 & $0.0225 / 112^{\circ}$ & $0.000577 / 111^{\circ}$ \\
\hline
\end{tabular}

En la Figura 7, se presentan los valores de los elementos discretos calculados para la red de adaptación, a través de la herramienta de Qucs. Los valores calculados son ideales por lo cual se deben realizar aproximaciones a valores comerciales. Para la mayoría de los elementos es posible obtenerlos en encapsulado 0201, en el caso del inductor L6 se debe convertir a elemento distribuido mediante la siguiente consideración:

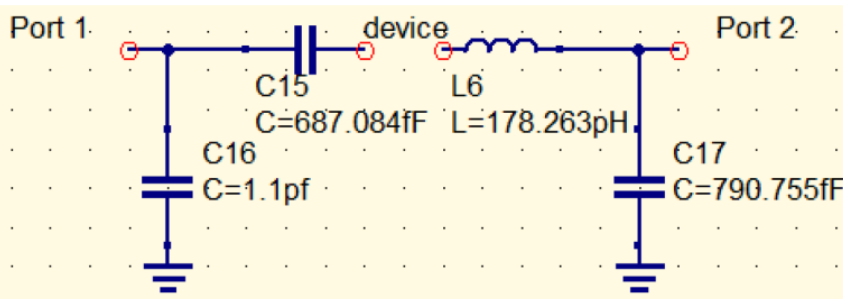

Figura 7. Red de adaptación puertos de entrada y salida 


$$
\begin{gathered}
X_{L}=\omega L \\
X_{L}=2 \pi\left(8.260 * 10^{9}\right)\left(178.263 * 10^{-12}\right)=9.25169 \Omega
\end{gathered}
$$

Se requiere que la línea de transmisión posea $50 \Omega$ en su parte real y $9.2516 \Omega$ en la imaginaria, entonces la impedancia quedaría definida de la siguiente manera:

$$
Z=50+j 9.25169=50.8487 \angle 10.483
$$

El siguiente paso es convertir el valor de impedancia en las dimensiones de una pista que puede ser sintetizada en el sustrato, el cálculo de esta línea depende de las características dieléctricas sobre la cual se va a implementar. Las dimensiones son: ancho $W=3.03 \mathrm{~mm}$, largo $L=0.6576 \mathrm{~mm}$.

Para la implementación final del amplificador se debe tener en cuenta consideraciones prácticas como las vías de interconexión a tierra de la capa superior a la inferior, si se tiene en cuenta que, una perforación de $1 \mathrm{~mm}$ en FR4 produce un efecto inductivo de $0.005 \mathrm{nH}$ a $0.2 \mathrm{nH}$ (Cripps, 2006). Para compensar este efecto se aplican tres perforaciones lo más cercanas posible al transistor y un capacitor en serie al pin de tierra de valor $6 p F$. Para referenciar adecuadamente la polarización del transistor se coloca una resistencia de $100 \Omega$ y por último se colocan líneas de transmisión de $50 \Omega$ y $\lambda / 4$ con el fin de acoplar los conectores de RF al circuito. En la Figura 8 se muestra el amplificador en su conjunto.

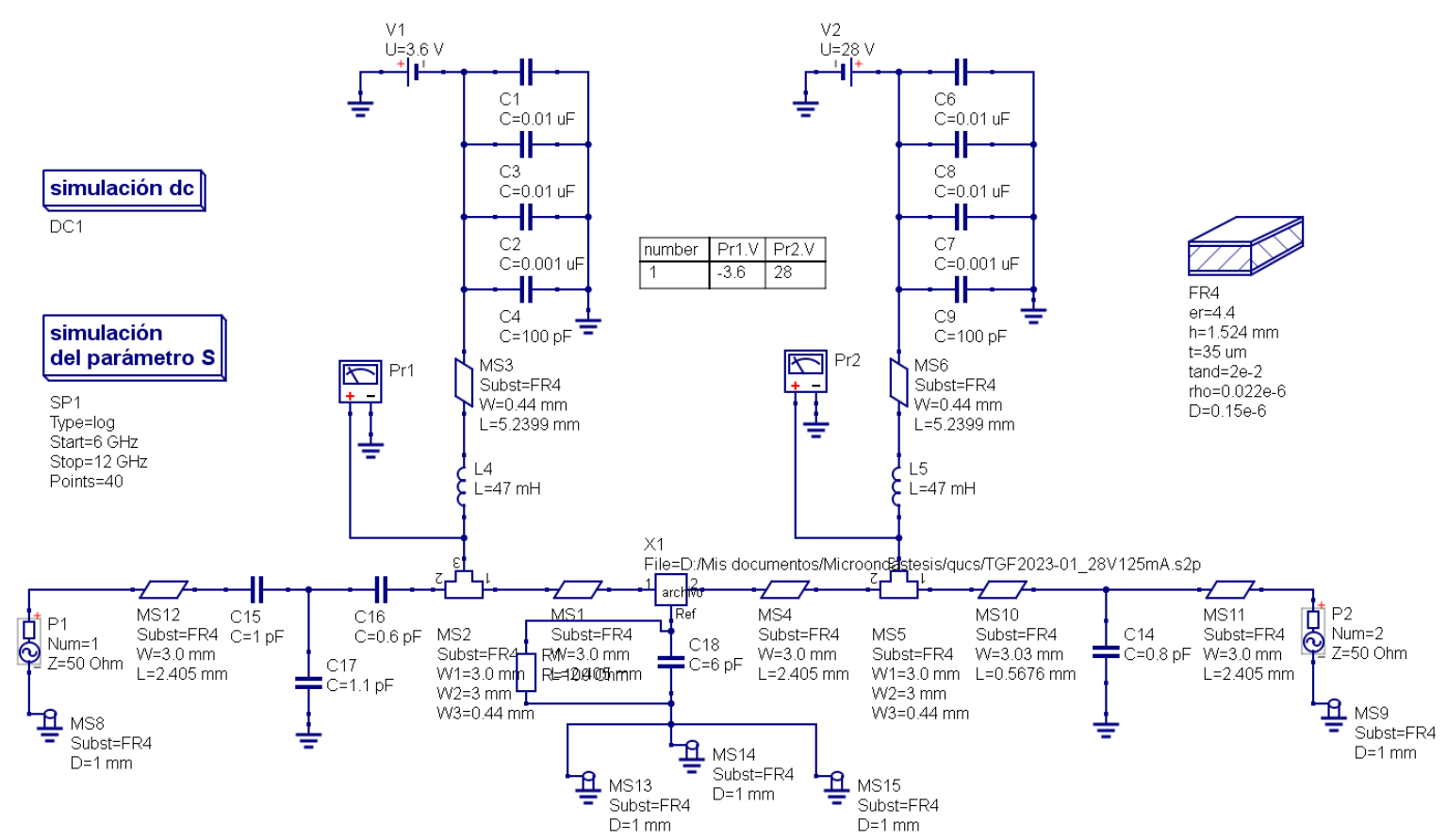

Figura 8. Red de adaptación puertos de entrada y salida

\section{Resultados y discusión}

\section{A. Ganancia de potencia}

Existen tres tipos de ganancias presentes en el amplificador además de la máxima ganancia disponible MAG. Estas son: la ganancia de transducción $G_{T}$ que es la relación entre la potencia en la carga $P_{L}$ y la potencia media de la fuente $P_{A V s}$ (ver Ecuación 8), ganancia de potencia $G_{P}$ que es la relación entre la potencia en la carga $P_{L}$ y la potencia 
de entrada $P_{I N}$ (ver Ecuación 9) y la ganancia disponible $G_{A}$ que es la relación entre la potencia media en la red $P_{A V n}$ y la potencia media en la fuente $P_{A V L}$, (ver Ecuación 10) (Zozaya, 2013).

La simulación se realiza en función de los parametros-S con barrido de frecuencia desde $6 \mathrm{GHz}$ a $12 \mathrm{GHz}$. En la Figura 9, se observa que $G_{T}, G_{P}$ y $G_{A}$ se mantienen estables con un valor de $14.6 \mathrm{~dB}$ en la frecuencia central de diseño, mientras que para los límites en el ancho de banda deseado la ganancia disminuye aproximadamente $1 d B$. En la Figura 10 se evalúa la máxima ganancia de potencia disponible que presenta un valor lineal para el rango de frecuencias en las cuales el dispositivo va operar, en esta figura tambien se incluye el valor de la estabilidad de Rollet con el cual se evalúa la oposicion del amplificador a oscilaciones cuyo valor es superior a 1 en todo el rango de simulación.

$$
\begin{gathered}
G_{T}=\frac{1-\left|\Gamma_{S}\right|^{2}}{\left|1-S_{11} \Gamma_{S}\right|^{2}}\left|S_{21}\right|^{2} \frac{1-\left|\Gamma_{L}\right|^{2}}{\left|1-S_{22} \Gamma_{L}\right|^{2}} \\
G_{P}=\frac{1}{1-\left|\Gamma_{I N}\right|^{2}}\left|S_{21}\right|^{2} \frac{1-\left|\Gamma_{L}\right|^{2}}{\left|1-S_{22} \Gamma_{L}\right|^{2}} \\
G_{A}=\frac{1-\left|\Gamma_{S}\right|^{2}}{\left|1-S_{11} \Gamma_{S}\right|^{2}}\left|S_{21}\right|^{2} \frac{1}{1-\left|\Gamma_{\text {OUT }}\right|^{2}}
\end{gathered}
$$

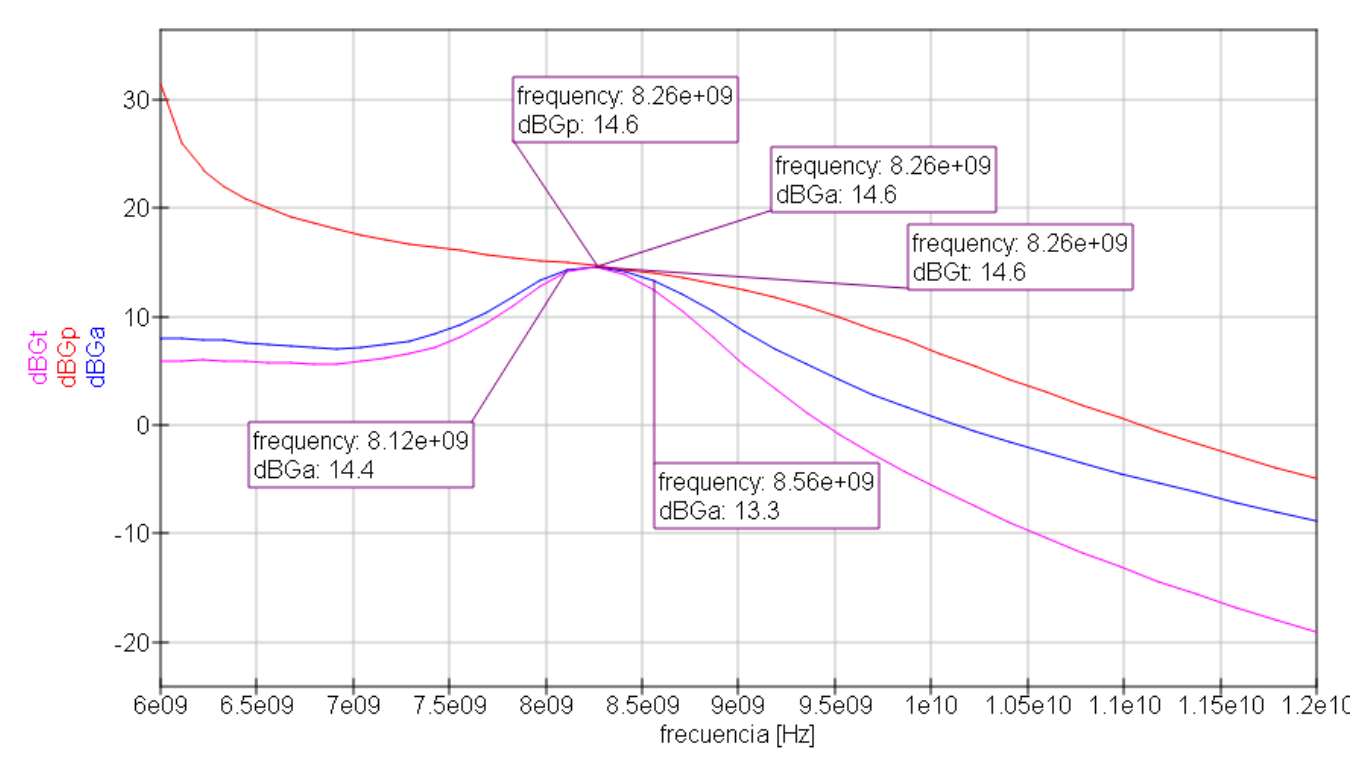

Figura 9. Niveles de ganancias presentes en el amplificador $G_{p}, G_{A}, G_{T}$

\section{B. Adaptación de puertos}

La adaptación de los puertos de entrada y salida se evalúa mediante un barrido de frecuencias desde $7 \mathrm{GHz}$ a $10 \mathrm{GHz}$ espaciados $0.13 \mathrm{GHz}$. En función de los parámetros-S, Qucs realiza la medición de impedancias en los puertos de entrada y salida y con funciones incluidas en el software de simulación se calculan los valores de coeficiente de reflexión y relación de onda estacionaria. En la Tabla 4 se muestran estos valores de los cuales se puede destacar que el puerto de salida presenta coeficientes de reflexión muy cercanos a cero en todo el rango de simulación, mientras que para el puerto de entrada está perfectamente adaptado en frecuencias cercanas a la de diseño. En la frecuencia central la relación es de 1:1 y en los límites del ancho de banda es de 1.1:1 referidos a $50 \Omega$. 


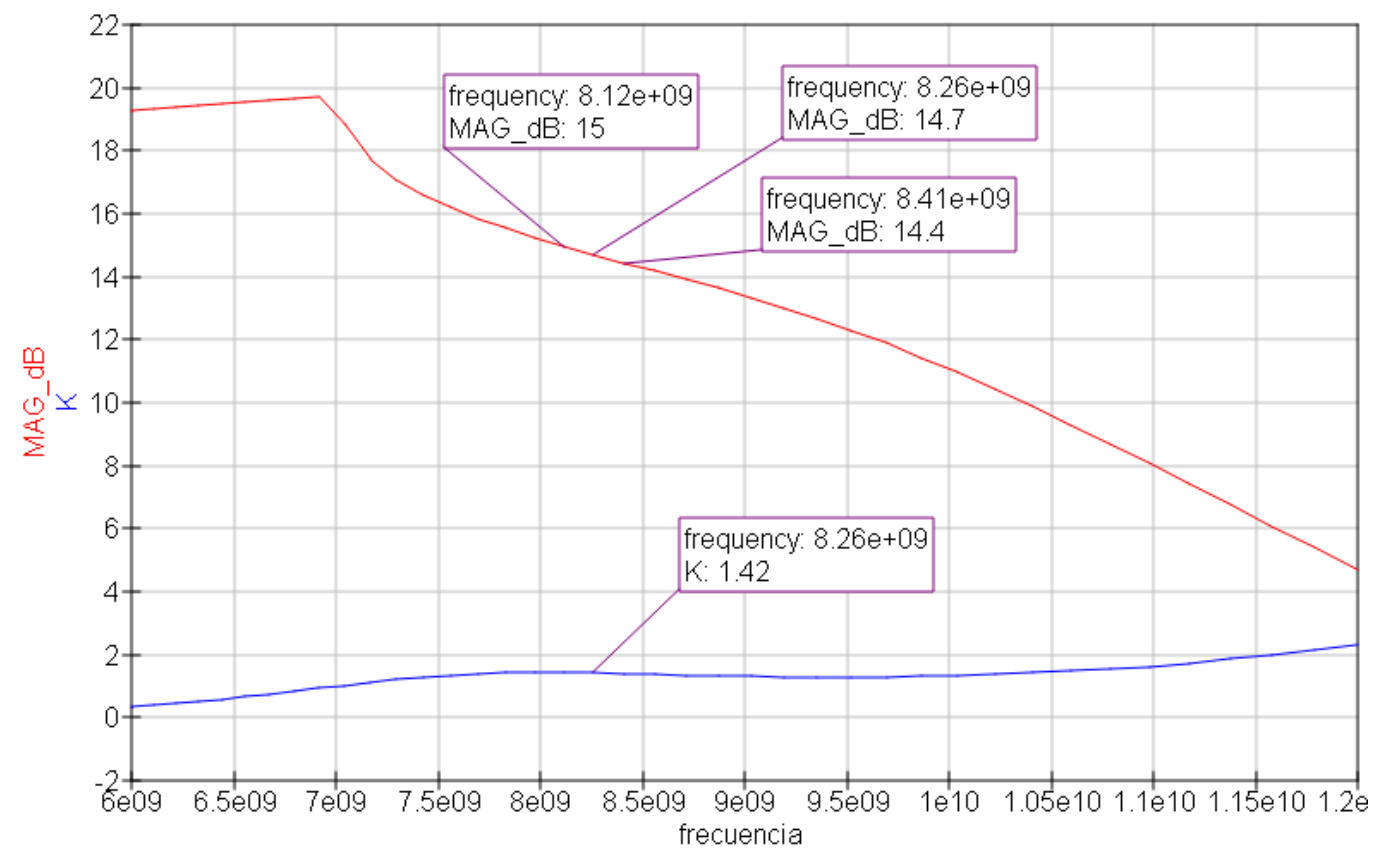

Figura 10. Máxima Ganancia de Potencia y factor de estabilidad de Rollet (K)

Tabla 4. Valores de adaptación de impedancias en puertos de entrada y salida

\begin{tabular}{|c|c|c|c|c|c|c|}
\hline Frecuencia [Hz] & $z_{s}[\Omega]$ & $z_{L}[\Omega]$ & $S W R_{\text {in }}$ & $S W R_{\text {out }}$ & $\Gamma_{s}$ & $\Gamma_{L}$ \\
\hline $7.040 \mathrm{E}+09$ & $27.1 / 37.4^{\circ}$ & $50.1 / 0.0291^{\circ}$ & 2.62 & 1.0 & $0.447 / 137^{\circ}$ & $0.000814 / 18.2^{\circ}$ \\
\hline $7.170 \mathrm{E}+09$ & $29.1 / 38.3^{\circ}$ & $50.1 / 0.0254^{\circ}$ & 2.54 & 1.0 & $0.435 / 133^{\circ}$ & $0.000566 / 23.1^{\circ}$ \\
\hline $7.300 \mathrm{E}+09$ & $31.4 / 38.6^{\circ}$ & $50 / 0.0265^{\circ}$ & 2.43 & 1.0 & $0.416 / 128^{\circ}$ & $0.000483 / 28.5^{\circ}$ \\
\hline $7.434 E+09$ & $3.44 / 37.6^{\circ}$ & $50 / 0.0288^{\circ}$ & 2.26 & 1.0 & $0.386 / 122^{\circ}$ & $0.000444 / 34.5^{\circ}$ \\
\hline $7.560 \mathrm{E}+09$ & $37.9 / 34.9^{\circ}$ & $50 / 0.032^{\circ}$ & 2.04 & 1.0 & $0.342 / 116^{\circ}$ & $0.060423 / 41.4^{\circ}$ \\
\hline $7.709 E+09$ & $42.1 / 30.0^{\circ}$ & $50 / 0.0355^{\circ}$ & 1.78 & 1.0 & $0.281 / 109^{\circ}$ & $0.000405 / 50^{\circ}$ \\
\hline $7.834 \mathrm{E}+09$ & $46.4 / 22.4^{\circ}$ & $50 / 0.0388^{\circ}$ & 1.5 & 1.0 & $0.202 / 101^{\circ}$ & $0.000383 / 62.1^{\circ}$ \\
\hline $7.970 \mathrm{E}+09$ & $49.6 / 12.6^{\circ}$ & $50 / 0.0436^{\circ}$ & 1.25 & 1.0 & $0.11 / 92.4^{\circ}$ & $0.000385 / 81.1^{\circ}$ \\
\hline $8.120 \mathrm{E}+09$ & $50.2 / 3.7^{\circ}$ & $50 / 0.0618^{\circ}$ & 1.07 & 1.0 & $0.0323 / 86.8^{\circ}$ & $0.000566 / 108^{\circ}$ \\
\hline $8.260 E+09$ & $49.9 / 0.328^{\circ}$ & $49.9 / 0.114^{\circ}$ & 1.01 & 1.0 & $0.00316 / 116^{\circ}$ & $0.00135 / 132^{\circ}$ \\
\hline $8.410 E+09$ & $48.5 / 0.244^{\circ}$ & $49.8 / 0.183^{\circ}$ & 1.03 & 1.01 & $0.0155 / 172^{\circ}$ & $0.00291 / 147^{\circ}$ \\
\hline $8.564 \mathrm{E}+09$ & $43.8 / 1.31^{\circ}$ & $49.6 / 0.212^{\circ}$ & 1.14 & 1.01 & $0.067 / 170^{\circ}$ & $0.004 / 153^{\circ}$ \\
\hline $8.711 \mathrm{E}+09$ & $40.3 / 4.32^{\circ}$ & $49.6 / 0.208^{\circ}$ & 1.26 & 1.01 & $0.114 / 161^{\circ}$ & $0.00403 / 153^{\circ}$ \\
\hline $8.870 E+09$ & $39.7 / 7.16^{\circ}$ & $49.7 / 0.2^{\circ}$ & 1.3 & 1.01 & $0.13 / 152^{\circ}$ & $0.00357 / 151^{\circ}$ \\
\hline $9.030 \mathrm{E}+09$ & $40.8 / 8.58^{\circ}$ & $49.7 / 0.194^{\circ}$ & 1.29 & 1.01 & $0.126 / 144^{\circ}$ & $0.00306 / 146^{\circ}$ \\
\hline $9.194 \mathrm{E}+09$ & $42.5 / 8.75^{\circ}$ & $49.8 / 0.192^{\circ}$ & 1.25 & 1.01 & $0.112 / 137^{\circ}$ & $0.00286 / 141^{\circ}$ \\
\hline $9.360 \mathrm{E}+09$ & $44.1 / 8.21^{\circ}$ & $49.8 / 0.19^{\circ}$ & 1.21 & 1.0 & $0.0952 / 131^{\circ}$ & $0.00233 / 135^{\circ}$ \\
\hline $9.520 \mathrm{E}+09$ & $45.6 / 7.33^{\circ}$ & $49.9 / 0.188^{\circ}$ & 1.17 & 1.0 & $0.0792 / 126^{\circ}$ & $0.00209 / 128^{\circ}$ \\
\hline $9.791 \mathrm{E}+09$ & $46.7 / 8.36^{\circ}$ & $49.9 / 0.186^{\circ}$ & 1.14 & 1.0 & $0.0651 / 121^{\circ}$ & $0.00191 / 122^{\circ}$ \\
\hline $9.870 \mathrm{E}+09$ & $47.6 / 5.43^{\circ}$ & $49.9 / 0.183^{\circ}$ & 1.11 & 1.0 & $0.0532 / 117^{\circ}$ & $0.00178 / 116^{\circ}$ \\
\hline $1.000 \mathrm{E}+10$ & $48.3 / 4.58^{\circ}$ & $49.9 / 0.18^{\circ}$ & 1.09 & 1.0 & $0.0434 / 113^{\circ}$ & $0.00167 / 110^{\circ}$ \\
\hline
\end{tabular}




\section{Conclusiones y recomendaciones}

En el artículo presentado se realiza un estudio de los métodos de diseño de un amplificador lineal de RF, el cual se optimiza para transmitir tonos puros en banda-X. Del trabajo presentado se destaca el ancho de banda de $500 \mathrm{MHz}$ alcanzado en el diseño propuesto. Además se logró obtener una ganancia de $14.6 \mathrm{~dB}$ mejorando así la estabilidad y la linealidad en la franja de frecuencias de trabajo del dispositivo activo. La estabilidad incondicional del transistor proporciona cierta libertad en la elección de los elementos que integran al amplificador final como elementos discretos y distribuidos, los resultados obtenidos cumplen con los requisitos planteados al inicio del trabajo.

El amplificador de RF clase A, brinda una topología simplificada y óptima para la transmisión de tonos puros. Esta topología en combinación con una adecuada elección del elemento activo reduce las etapas necesarias para lograr la máxima transferencia de potencia. El diseño de las redes de adaptación de impedancias para los puertos de entrada y salida del amplificador mejoran la estabilidad de Rollet $(K>1)$ en el rango de frecuencias que va a operar y ofrece adaptación en ambos puertos de 1:1.

Se recomienda a los diseñadores realizar un estudio previo de las características del transistor en el rango de frecuencias específicas en que va a operar mediante sus parámetros-S. Este procedimiento ayuda a disminuir la complejidad en el diseño de las redes de adaptación. La inclusión del efecto del dieléctrico y las perforaciones necesarias para las pistas del circuito, desde la primera etapa de diseño, disminuye las iteraciones en el proceso de la elaboración final del dispositivo.

\section{Bibliografía}

Cripps, S. C. (2006). RF power amplifiers for wireless communications (Segunda ed.). Norwood: Artech House microwave.

Froehner, W. H. (1967). Quick amplifier design with scattering parameters. S-Parameters, circuit analysis and design, 5,1-5,11.

Glover, I. A., Penoock, S. R., \& Shepherd, P. R. (2005). Microwave Devices, Circuits and Subsistems for Comunications Engineering. West Sussex: John Wiley \& Sons, Ltd.

Hu, L., Qian, K., \& Wang, B. (2015). Research of High Efficiency X-Band Power Amplifier. (IEEE, Ed.) International Symposium on Computational Intelligence and Design, 129-132. doi:10.1109/ISCID.2015.214

Instituto Espacial Ecuatoriano. (12 de noviembre de 2016). Instituto Espacial Ecuatoriano. Obtenido de http://www.institutoespacial.gob.ec/

Köprü, R., Kuntman , H., \& Yarman, B. (2013). A Novel Method to Design Wideband Power. IEEE, 1942-1945.

Pérez, J. C., \& Jaramillo, I. J. (2012). Methodology of design and construction of microwave power amplifiers prototypes for uhf radio frequency band. Bogotá D.C., Colombia: Universidad Nacional de Colombia.

Qucs. (12 de mayo de 2017). Quite universal circuit simulator. Obtenido de http://qucs.sourceforge.net/docs.html

Resca, D., Raffo, A., Falco, S. D., Scappaviva, F., Vadalà, V., \& Vannini, G. (Abril de 2014). X-Band GaN Power Amplifier for Future Generation SAR Systems. IEEE MICROWAVE AND WIRELESS COMPONENTS LETTERS, 24(4), 266-268. doi:10.1109/LMWC.2014.2299552

Sayre, C. W. (2001). Complete Wireless Design (Segunda ed.). New York: McGraw-Hill.

Setiawan, E., Latin, M., \& Alam, B. (2017). Design and Simulation of Front-End Broadband RF Power. International Symposium on Electronics and Smart Devices, 314-317.

Smith, R., \& Cripps, S. (2016). Broadband Push-Pull Power Amplifier Design at. Proceedings of the 46th European Microwave Conference, 1353-1356. 
Soler, J. J. (2015). Diseño de un amplificador clase $F$ inverso a $3.5 \mathrm{GHZ}$ usando tecnología GaN-HEMT. Ingeniería, negocios e innovación, 33-44.

Suárez, A., Wentzel, A., \& Heinrich, W. (2017). Stability Analysis of Digital Microwave. IEEE TRANSACTIONS ON MICROWAVE THEORY AND TECHNIQUES, 30563070.

Virdee, B. S., Virdee, A. S., \& Banyamin, B. Y. (2014). Broadband Microwave Amplifiers. London: Artech House.

Yarman, B. S., \& Ejaz, M. (2015). Practical Consideration to Design Broadband. Microwave Symposium (MMS, IEEE 15th Mediterranean, 1-4).

Zozaya, A. J. (2013). Diseño de amplificadores lineales y de bajo ruido de RF (Tema 2). Carabobo: Universidad de Carabobo. 